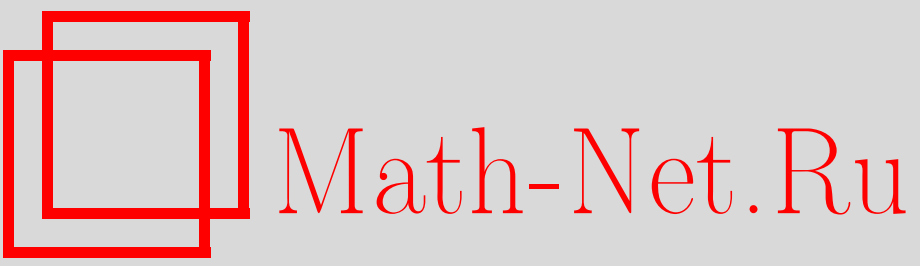

С. В. Людковский, Квазиинвариантные меры на неархимедовых банаховых пространствах, УМН, 2003, том 58, выпуск 2, 167-168

DOI: https://doi.org/10.4213/rm619

Использование Общероссийского математического портала Math-Net.Ru подразумевает, что вы прочитали и согласны с пользовательским соглашением

http://www.mathnet.ru/rus/agreement

Параметры загрузки:

IP: 54.224 .187 .69

26 апреля 2023 г., 06:42:47 


\section{КВАЗИИНВАРИАНТНЫЕ МЕРЫ НА НЕАРХИМЕДОВЫХ БАНАХОВЫХ ПРОСТРАНСТВАХ}

\section{С.В. Людковский}

Имеется ряд работ об интегрировании в банаховых пространствах (БП) и квазиинвариантных мерах в классическом случае (т.е. для БП над полями $\mathbb{R}$ или $\mathbb{C}$ ), тогда как для неархимедовых БП $X$ (т.е. для БП над неархимедовыми полями $\mathbb{K}$ ) критерии квазиинвариантности мер (КИМ) относительно линейных и нелинейных преобразований БП $X$ не рассматривались. С другой стороны, развитие неархимедовой квантовой механики и неархимедова функционального анализа приводит к необходимости решения таких проблем, чему и посвящена данная работа (см. также [1]).

1. Замечания и определения. Для хаусдорфова топологического пространства $X$ с малой индуктивной размерностью ind $(X)=0$ борелевская $\sigma$-алгебра обозначается $\mathscr{B}$. Далее действителшные меры $\mu$ задаются на измеримых пространствах $(X, F)$, где $\sigma$-алгебра $F \supset \mathscr{B}$.

Напомним, что для алгебры подмножеств $E$ в $X$, разделяющей точки в $X$, отображение $\mu$ из $E$ в неархимедово поле $\mathbb{K}_{s}$ такое, что оно полно как ультраметрическое пространство и $\mathbb{Q}_{s} \subset \mathbb{K}_{s} \subset \mathbb{C}_{s}$, назьвается мерой, если выполнены следующие условия: (i) $\mu$ аддитивна и $\mu(\varnothing)=0$, (ii) для любого $A \in E$ существует норма $\|A\|_{\mu}:=\sup \left\{|\mu(B)|_{\mathbb{K}_{s}}: B \subset A\right.$, $B \in E\}<\infty$, (iii) если имеется сжимающееся семейство $F$, т.е. для любых $A, B \in F$ существует $F \ni C \subset A \cap B$ и $\bigcap\{A: A \in F\}=\varnothing$, то $\lim _{A \in F} \mu(A)=0$ (см. обозначения в [2] и там же о пополнении $A f(X, \mu)$ алгебры $E$ по мере $\mu$ ). Меру со значениями в $\mathbb{K}_{s}$ назовем вероятностной, если $\|X\|_{\mu}=1$ и $\mu(X)=1$. Для функций $f: X \rightarrow \mathbb{K}_{s}$ и $\phi: X \rightarrow[0, \infty)$ используется обозначение $\|f\|_{\phi}:=\sup _{x \in X}(|f(x)| \phi(x)), N_{\mu}(x):=\inf \left(\|U\|_{\mu}: U \in E, x \in X\right)$. Плотные меры, т.е. определенные на $E \supset \mathrm{Bco}(X)$, образуют БП $M(X)$ с нормой $\|\mu\|:=\|X\|_{\mu}$, где $\mathrm{B} \operatorname{co}(X)-$ алгебра открыто-замкнутых подмножеств в $X$.

Пусть БП $X$ задано над бесконечным полем $\mathbb{K}$ с дискретной нетривиальной группой $\Gamma_{\mathbb{K}}:=\left\{|x|_{\mathbb{K}}: x \neq 0\right\}$. Пусть имеется возрастающая последовательность БП $L_{n} \subset L_{n+1} \subset \ldots$ такая, что $\operatorname{cl}\left(\bigcup\left[L_{n}: n\right]\right)=X, \operatorname{dim}_{\mathbb{K}} L_{n}=\kappa_{n}<\aleph_{0}$ для всех $n$. Выберем согласованное семейство проекций $P_{n}^{m}: L_{m} \rightarrow L_{n}$ для любых $m \geqslant n$, тогда согласованное семейство мер $\mu_{L_{n}}(A)=\mu_{L_{m}}\left(P_{n}^{-1}(A) \cap L_{m}\right)$ при любых $m \geqslant n$ называется последовательностью конечномерных распределений.

2. ОПредЕлЕниЕ. Пусть на $X$ заданы две ненулевые меры $\mu$ и $\nu$ со значениями в $\mathbb{R}$ или $\mathbb{K}_{s}$. Мера $\nu$ назьвается абсолютно непрерывной относительно меры $\mu$, если $\nu(A)=0$ для любого $A \in E$ с $\mu(A)=0$, или для любого $A \in E: \nu(A)=\int_{A} f(x) \mu(d x)$, где $f \in L(\mu)$, что обозначается $\nu \ll \mu$. Если $\nu \ll \mu$ и $\mu \ll \nu$, то они называются эквивалентными, $\nu \sim \mu$.

3. ТеОРема. Пусть $X=\prod_{j=1}^{\infty} X_{j}$ - произведение вполне регулярных пространств $c \operatorname{ind}\left(X_{j}\right)=0$ и вероятностныци мерами $\mu_{j}, \nu_{j}: E\left(X_{j}\right) \rightarrow \mathbb{K}_{s}, a \mu_{j} \ll \nu_{j}$ для лю-

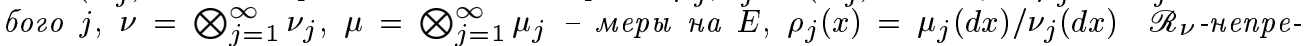
рывны по $x \in X_{j}, \prod_{j=1}^{n} \rho_{j}\left(x_{j}\right)=: t_{n}(x)$ сходится равномерно на $A f(X, \nu)$-компактных подмножсествах в $X, \beta_{j}:=\left\|\rho_{j}(x)\right\|_{\phi_{j}}, \phi_{j}(x):=N_{\nu_{j}}(x)$ на $X_{j}$. Eсли $\prod_{j=1}^{\infty} \beta_{j}$ сходится в $(0, \infty)$ (или расходится $\kappa 0)$, то $\mu \ll \nu u q_{n}(x)=\prod_{j=1}^{n} \rho_{j}\left(x_{j}\right)$ сходится в $L(\nu) \kappa q(x)=\prod_{j=1}^{\infty} \rho_{j}\left(x_{j}\right)=\mu(d x) / \nu(d x)($ или $\mu \perp \nu$, m.e. cyществует $F \in E c$ $\left.\|F\|_{\mu}+\|X \backslash F\|_{\nu}=0\right)$, zде $x_{j} \in X_{j}, x \in X$.

4. ОПРеДЕЛЕНИЕ. Для БП $X$ над $\mathbb{K}$ элемент $a \in X$ называется допустимым сдвигом меры $\mu$ со значениями в $\mathbb{R}$ или $\mathbb{K}_{s}$, если $\mu_{a} \ll \mu$, где $\mu_{a}(A)=\mu(A-a)$ для любых $A \in E, \rho(a, x):=$ $\rho_{\mu}(a, x):=\mu_{a}(d x) / \mu(d x), M_{\mu}:=\left[a \in X: \mu_{a} \ll \mu\right]$. Мера $\mu$ назьвается квазиинвариантной, если $M_{\mu}$ содержит $\mathbb{K}$-линейное подпространство $J_{\mu}$, плотное в $X$.

5. Теорема. Если $\mu: E \rightarrow \mathbb{R}(\bigvee \mathbb{F})$ - б-конечная мера на $E(Y)($ или $\mathrm{B} c о(Y)), Y$ полное сепарабельное ультраметрическое $\mathbb{K}$-линейное пространство такое, что со $(S)$ 
нигде не плотно в $Y$ для любого компакта $S \subset Y$, где $\mathbb{K} u \mathbb{F}$ - бесконечные недискретные нерхимедовы поля. Тогда из $J_{\mu}=Y$ следует, что $\mu=0$.

6. Теорема. Пусть $X$ - сепарабельное БП над локально компактныц полем $\mathbb{K}$. Тогда существуют вероятностные КИМ н на $X$ со значениями в $\mathbb{R}$ или $\mathbb{K}_{s}(s \neq p)$.

7. ЗАмечАнИЕ. Пусть $X-$ БП над $\mathbb{K},|*|_{\mathbb{K}}=\bmod _{\mathbb{K}}(*)$ с вероятностной КИМ $\mu: E \rightarrow \mathbb{R}$ $\left(\vee \mathbb{K}_{s}\right)$ и $\mu_{L_{n}}(d x)=f_{n}(x) v_{n}(d x)$, где $f_{n} \in L^{1}\left(L_{n}, v_{n}, \mathbb{R}\right)\left(\vee L\left(L_{n}, v_{n}, \mathbb{K}_{s}\right)\right), v_{n}(a+A)=$ $v_{n}(A)$ для любых $a \in L_{n}$ и $A \in E\left(L_{n}\right)$, а оператор $U \mu$-почти всюду на $X$ удовлетворяет следующим условиям: (i) $U \in C^{1}$; (ii) $\left(U^{\prime}(x)-I\right)$ компактен; (iii) $(x-U(x)) \in J_{\mu}$; (iv) $(x-U(x) ; x) \in$ $\operatorname{dom}(\rho(z, x))$, причем $\rho(x-U(x), x) \neq 0(\bmod \mu) ;(\mathrm{v}) \mu\left(S^{\prime}\right)=1\left(\vee\left\|X \backslash S^{\prime}\right\|_{\mu}=0\right)$, здесь $S^{\prime}:=([x: \rho(z, x)$ определена для $z \in L$ и непрерьвна по $z])$ для любого конечномерного $L \subset J_{\mu}$ над $\mathbb{K} ;(\mathrm{vi})$ существует $S$ с $\mu(S)=0\left(\vee\|S\|_{\mu}=0\right)$ и для любого $x \in X \backslash S$ и любого $z$, для которого существует $\rho(z, x)$, выполнено условие: $\lim _{n \rightarrow \infty} \rho\left(P_{n} z, x\right)=\rho(z, x)$ и сходимость равномерна для любого конечномерного над $\mathbb{K}$ подпространства $L \subset J_{\mu}$ по $z$ в $L \cap\left[x \in J_{\mu}:|x| \leqslant c\right]$, где $c>0$; (vii) существует $n$, для которого при всех $j>n$ и $\mu$-почти всех $x \in X$ отображения $U(j, x):=x+P_{j}(U(x)-x)$ обратимы и $\lim _{j}\left|\operatorname{det} U^{\prime}(j, x)\right|=\left|\operatorname{det} U^{\prime}(x)\right|$ (дополнительно с равномерной сходимостью в (vi) по $z$ на $A f(X, \mu)$-компактных подмножествах для $\rho\left(P_{n} z, x\right)$, а в (vii) по $x$ для $\left|\operatorname{det} U^{\prime}(j, x)\right|$ для $\mu$ со значениями в $\left.\mathbb{K}_{s}\right)$. Тем же условиям пусть удовлетворяет $U^{-1}$.

8. ТЕорема. Из предположений $n .7$ следует, что мера $\nu(A):=\mu\left(U^{-1}(A)\right)$ әквивалентна $\mu$ и $\nu(d x) / \mu(d x)=\left|\operatorname{det} U^{\prime}\left(U^{-1}(x)\right)\right|_{\mathbb{K}} \rho\left(x-U^{-1}(x), x\right)$.

\section{СПИСОК ЛИТЕРАТУРЫ}

[1] S. V. Ludkovsky. Quasi-invariant and pseudo-differentiable measures on a non-Archimedean Banach space // Los Alamos Nat. Lab. USA. math. GM/0106169 и math . GM/0106170 (ICTP: IC/96/210). [2] A. C. M. van Rooij. Non-Archimedean Functional Analysis. New York: Dekker, 1978. 